\title{
Exploring Personalized Travel Route Using POIs
}

\author{
Jie Li, Yujiu Yang, and Wenhuang Liu
}

\begin{abstract}
The development of travel industry gradually fosters personalized requirements of tourists, such as setting the start place, exploiting interesting activities and organizing the travel route. In this paper, we take advantage of two online websites to collect information of both scenic spots and real-world local activities, and we proposed three rule-based methods to recommend travel routes for tourists, which can take their instant location into account and satisfy their personalized demands. Specifically, we extract scenery spots dataset from Flickr and popular activities from Douban-Event in line with the visiting city and date. Then we process these items and take the filtering results as the recommendation candidates. Finally based on popularity and geographical location of these venues/activities, we focus on arranging a colorful travel route, which covers not only the everlasting scenic spots, but also some temporary activities. We evaluate the quality of travel routes produced by our recommendation approaches with an alternative plan suggested by a professional travel site. The results justify the advantages of our approaches over the baseline method provided by travel experts.
\end{abstract}

Index Terms-DoubanEvent, flickr, popularity, travel route.

\section{INTRODUCTION}

Even though there are numerous tourism websites and travel agencies to provide various travel packages, tourists just become puzzled about how to make a choice and neither could they adjust the travel plan. Besides, if tourists try to arrange the travel route by themselves, tremendous information is easy to exhaust them when considering the location interest, visiting time, price, etc. So it is desirable if a travel recommender could help a tourist to find places matching his interests and his current situation.

There are some unique characteristics of travel data. First the dataset is very sparse [1]. Compared with the traditional recommendation data (such as MovieLens and Netflix), there are so few co-traveling records that it is troublesome to find credible nearest neighbors for the specific user, which increases the difficulties for employing collaborative filtering skills. Second, each attraction/activity has a physical location It is not convenient or economical for tourists to spend much time on transportation, so we need to take travel distance into account and the attractions/activities nearby are more suitable to constitute the same recommendation travel route. Third, unlike traditional recommendation where the price of an item is not a concern, in travel recommendation financial cost is a vital consideration, which means that a bad recommendation

Manuscript received February10, 2014; revised May 2, 2014.

Jie Li, Yujiu Yang, and Wenhuang Liu are with Graduate School at Shenzhen, Tsinghua University, China (e-mail: jli11@tsinghua.edu.cn, yang.yujiu@sz.tsinghua.edu.cn, lwh@sz.tsinghua.edu.cn). not only wastes the user's time but also his money a lot. Fourth, long tail effect exists in this field too and [2], [3] also implicate that tourists sometimes attend popular local events regardless of their interests. This signifies it is necessary to calculate popularity and make travel suggestion based on it. Finally, LDA (Latent Dirichlet Allocation) is often used to infer the user interest and local preference, but if data is sparse or all the users are new (cold start problem), there is no travel history to estimate the personal interest and it is harder to make accurate and meaningful recommendation using LDA. Flickr is one of the best online photo management and sharing websites in the world. [1], [4]-[6] take advantage of Flickr data to make travel recommendation. DoubanEvent is the largest event-based social networking site in China which provides multiple information about local activities, including time, address, price and the number of participants [3]. From the two websites, we get the specific longitude and latitude of the place and define the popularity [7] of events and scenic spots. For simplicity, we utilize POIs (Point of Interest) to stand for both scenic spots and events.

In this paper, we aim to offer the user a travel route covering a set of POIs (scenic spots or events) by giving consideration to both popularity and distance. Three approaches are proposed: Distance-based recommendation (DR), Popularity-based recommendation (PR) and Distance-Popularity-based recommendation (DPR). The methods are designed to be deployed in an application scenario that is suitable for everyone, without limitation to the tourists who already have travel history in the given dataset or have been the registered members of some specific website. Moreover, it could also match the user's personalized demands, such as selecting the travel city/date and setting the start point for the route. To the best of our knowledge, this is the first work that local activities information is included to construct the travel route. Our contributions in this article are summarized as follows:

- Popularity: in order to define the level of a POI being interested by users quantitatively, we adopt the concept of popularity and apply a new definition of it, which considers not merely the number of unique visits made to those POIs [5] but also the number of views/comments/favorites produced on the website. And we make use of the popularity to filter the locations and then rank the POIs in PR/DPR method.

- Activity/event: the travel route incorporates both scenic spots and temporal activities. The common travel recommendation only takes attractions into account, which could be visited almost at any time. In our opinions, attending some popular online activities is a good way to understand local history and culture. If the travel recommender includes such activities, users would be interested in them and obtain benefits. The research 
survey verifies our assumption.

\section{PRELIMINARY}

\section{A. Problem Statement}

We define our problem as follows: given a user u planning to visit a city $C u$ at the day $D u$ with regarding the place $L o$ as the start point, which POIs should he go? What is the sequence between these POIs?

This problem poses several challenges: 1) find a free data source of scenic spots/activities and extract available recommendation items; 2) choose the scenic spots/activities to match user's demands and interests; 3) organize the selected items so that the user could visit them conveniently

\section{B. Definition}

Definition 1. (Popularity) In order to determine the level of a POI being interested by users we decide that each POI should include a notion of popularity. For scenic spots extracted from Flickr, we gather all the photos referring to the same place, and aggregate the frequencies to describe scenic spots respectively. Compared with other definitions [4], [7], our definition considers more related factors so it is more objective and reasonable. The popularity of a POI (scenic spot) is calculated as.

$$
P O P=a_{1} N_{v}+a_{2} N_{f}+a_{3} N_{c}+a_{4} N_{u}
$$

where the coefficients are set: $a_{1}=0.01, a_{2}=0.1, a_{3}=0.5$, $a_{4}=1 . N_{v}, N_{f}, N_{c}, N_{u}$ mean the number of views, favorites, comments and users belonging to the specific scenic spot respectively.

Let's take "Window of the World" as an example. There are 4,115 photos taken at this place and uploaded to Flickr. Through adding up these photos, we get:

$$
N_{v}=66382, N_{f}=196, N_{c}=79, N_{u}=305
$$

So the popularity of "Window of the World" will be set to: $P O P=0.01 \times 66382+0.1 \times 196+0.5 \times 79+1 \times 305=1074.72$. For social activities extracted from DoubanEvent, there are two buttons on the page of activity, which are participant and wisher. The user can express his opinion by clicking the button, and we make use of the two factors to calculate the popularity of an activity. The detailed is showed as (1).

$$
P O P=a_{1} N_{p}+a_{2} N_{w}
$$

Coefficients are set: $a_{1}=1, a_{2}=0.5 . N_{p}, N_{w}$ mean the number of participants and wishers of the activity respectively. For instance, there is a drama, and 527 users indicate to attend it and 1140 users just have interests. So the popularity of this drama will be set to:

$$
P O P=1 \times 527+0.5 \times 1140=1097
$$

Trough above definition, we still need to normalize the two popularities so that the value of popularity will locate in the range of $[0,1]$. Eq.(3) has been adopted. If the POI is a scenic spot, Min and Max stand for the Minimum and Maximum value of the scenic spot's popularity; if the POI is an activity, Min and Max stand for the Minimum and Maximum popularity value of the activity.

$$
P O P_{n e w}=\left(P O P_{\text {old }}-\operatorname{Min}+t\right) /(\operatorname{Max}-\operatorname{Min}+t)
$$

Definition 2. (Distance) Tourists prefer visit a bunch of nearby POIs [1], so it is meaningful to define the distance between POIs. At first we collect the longitude and latitude of POIs and then we use (4) to calculate the distance between

$$
\begin{gathered}
\text { poi }_{1}\left(\operatorname{lat}_{1}, \operatorname{lng}_{1}\right) \text { and } \text { poi }_{2}\left(l a t_{2}, \operatorname{lng} g_{2}\right) . \\
\text { Dis }=2 \arcsin \left(\sqrt{\sin \left(\frac{a}{2}\right)^{2}+\cos r_{1} \cdot \cos r_{2} \cdot \sin \left(\frac{b}{2}\right)^{2}}\right) \\
r_{1}=\operatorname{rad}\left(l a t_{1}\right) \quad r_{2}=\operatorname{rad}\left(l a t_{2}\right) \\
a=r_{1}-r_{2} \quad b=\operatorname{rad}\left(\operatorname{lng}_{1}\right)-\operatorname{rad}\left(\operatorname{lng}_{2}\right)
\end{gathered}
$$

\section{RECOMMENDATION ALGORITHM}

\section{A. Framework}

The architecture behind our approach is depicted in Fig. 1. It is configured into various modular tasks. We give an overview of these operations here and details are presented in the following sections.

1) Tourist input the travel requirements, including the visiting city, visiting date and the start point;

2) Collect scenic spots metadata based on the city and activities metadata based on the city and date;

3) Calculate popularity of POI and extract name \& geo-coordinates from the POI metadata;

4) Recommend travel route based on popularity and distance.

From Fig. 1, we can understand the process of recommendation. At first, the tourist tells our system which city he wants to visit, the time he wants to come and the place he wants to set as the beginning point. The start point can be determined by the user's manual input or delivered by GPS module embedded in his smart phone. After receiving these initial requirements, the system collects the POI information. Since the number of cities is limited and the scenic spots information would not change frequently, so the scenic spots metadata of cities have been collected offline. However, activities changed every day, which means there are different activities in a separate day. So the system extracts activities information from DoubanEvent online. Then according to (1), (2), (3), the system calculates the popularity of each POI, which makes up POI database with other two attributes (name and geo-coordinates). With these preparations, the system could provide travel routes for the user. The detailed recommendation algorithm will be explained in the following sections.

\section{B. Algorithm}

To recommend personalized travel route, we propose three rule-based approaches: Distance-based Recommendation (DR), Popularity-based Recommendation (PR) and 
Distance-Popularity-based Recommendation (DPR). The requirements of the three Algorithms are similar and are given a united explanation here.

\section{1) Requirements}

The traveling city $C$; the traveling date $D ; P O I=\left(p o i_{1}\right.$, poi $_{2}, \ldots$, poi $\left._{n}\right) ;$ poi $_{i}=\left(i_{i}\right.$, name $_{i}$, lat $_{i}, \operatorname{lng}_{i}$, pop $_{i}$ ) ; start point $l_{0}=\left(l a t_{0}, \ln g_{0}\right)$; the number of visiting POIs in a day $k=7$ here. POI is a finite set of POIs related to user's input (city $C$, date $D$ )

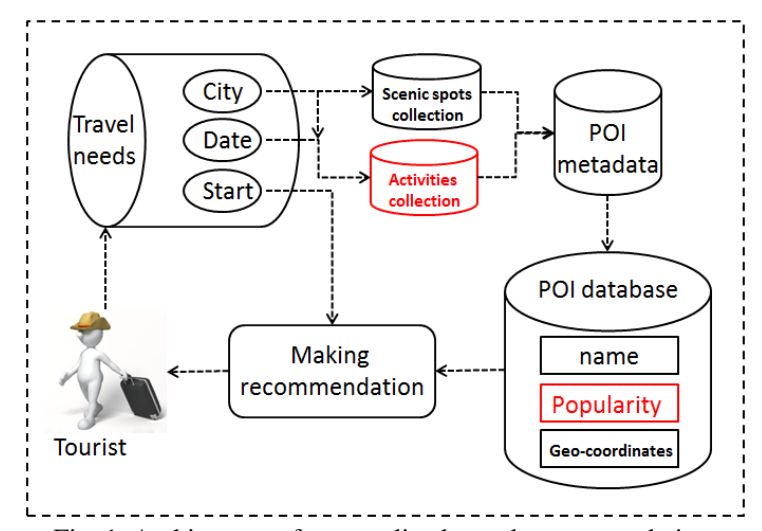

Fig. 1. Architecture of personalized travel recommendation.

\section{2) Distance-based recommendation (DR)}

First, based on the geographic position of start point denoted as $l_{0}\left(l a t_{0}, \ln g_{0}\right)$, the system search for the POI geo-coordinates from POI database and calculate the distance as (4) between every candidate POI and the start point. Then the system ranks all the distance and find out the POI that produces the minimum distance as the next destination denoted as $l_{1}\left(l a t_{1}, l n g_{1}\right)$. The POIs that have been arranged in the travel route will not be considered in the following calculation. This process is repeated until receiving 7 traveling POIs and the sequence between the POIs is also determined accordingly. Through the above description, we can see that only the geo-coordinates are used and the distances between the POIs are counted, so we name this approach Distance-based Recommendation. Besides, the start point is designated by the user and it would influence the recommendation result tremendously because different start point will get different nearest POI and also affect the following calculation. Algorithm 1 describes DR method and Fig. 2(a) depicts the recommendation route using this method.

\section{3) Popularity-based recommendation (PR)}

First, we fetch the popularity of all the POIs of the target city and rank them. Then the system picks out the top seven POIs as the candidates of recommended travel route. After that, based on the start point, we arrange the sequence of the seven

\section{EXPERIMENT}

POIs and the processing method is similar to the method mentioned in DR. From the seven POIs, we get the POI closest to the start point as the first visiting point. And the rest POI can be arranged in the same manner. If adopting this approach, the candidate POIs will not change but the visiting order will be adjusted to the requirement of the user. As popularity of POI is the most important fact to be taken into consideration, so we name this approach Popularity-based Recommendation. Fig. 2(b) depicts the recommendation route using this method.
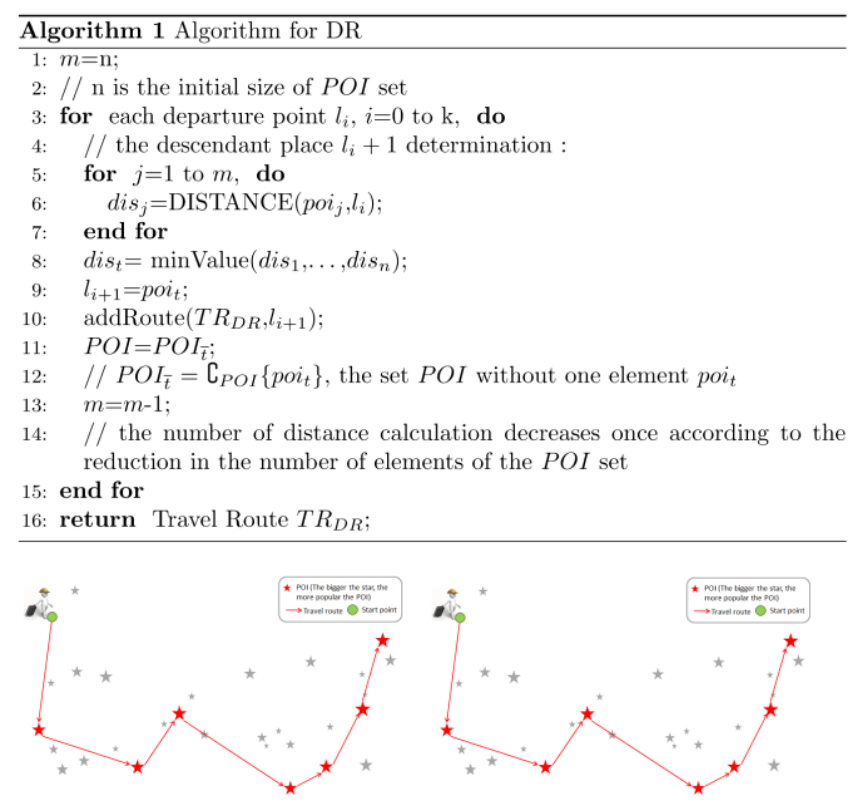

(a) DR approach

(b) PR approach

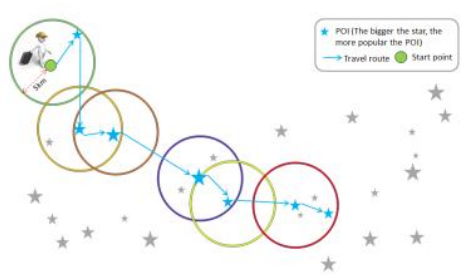

(c) DPR approach

Fig. 2. Recommended travel route.

\section{A. Distance-Popularity-Based Recommendation (DPR)}

This approach combines the distance with popularity and is relatively complex compared with the above two methods. First, the system calculates the distance between each existing POI and the start point. Set $5000 \mathrm{~m}$ as a threshold, which means if there is no any POI within $5000 \mathrm{~m}$ then we pick out the nearest POI from the entire dataset as the next destination. Otherwise, we collect all the POIs within $5000 \mathrm{~m}$, and compare their popularities. The POI with the highest popularity will be set as the next visiting point. Every point is determined one by one according to this rule until collecting seven POIs. Because this approach incorporates both popularity and distance, so we name it Distance-Popularity-based Recommendation. Fig. 2(c) depicts the recommendation route using this method.

\section{B. Dataset}

Unlike the traditional recommendation, some datasets are publicly released and extensively used, such as MovieLens and Netflix Prize Dataset, in travel recommendation, no open web sources provide available travel information. [8] uses two GPS trajectory datasets generated by over 12,000 
taxicabs in Beijing and two POI datasets of Beijing. In order to discover the tourists' travel motivations, [9] considers 30,000 questionnaires completed by tourists between 2001 and 2009. Our work is also facing such problem, so we collect the data by ourselves.

Flickr dataset: The Flickr database is open to everyone via the FlickrAPI, which allows users to search Flickr photo databases for geotagged images [4]. At the present stage, we only consider the city of Shenzhen, which means tourists plan to visit Shenzhen and we give POIs recommendation in Shenzhen. It is easy to generalize to other cities.

First, using the Flickr API, we gathered all the photos about Shenzhen and all the metadata are extracted from these photos. The detailed information about these photos is showed as TABLE I. We also discover the power law distributions of attractions. Some popular landmarks are traveled by many tourists and other unknown destinations only have a few photos. We discard some destinations if the number of these photos is less than five. After filtering, we got twenty-nine scenic spots of Shenzhen and the number of photos is demonstrated as Fig. 3. The name of scenic spots corresponding to id is demonstrated in Appendix A.

DoubanEvent dataset: DoubanEvent is the largest social networking site in China where users can publish and participate in social activities. On DoubanEvent, an activity is presented by a user with specifying the date/address/content/ price/type of the activity. Other users can sign up or express interest in this activity online. When the tourist tells the system he will visit city $C u$ at the day $D u$, the travel recommendation system will use a crawler to retrieve the original information (see TABLE II) in the city at that day from DoubanEvent website.

\section{Experiment Design}

We evaluate the quality of travel routes produced by different recommendation techniques. Through the user survey, we prove that our approaches outperform the professional tour website comprehensively. Furthermore, we show that the distance-based recommendation gives a more satisfying result than PR and DPR, which is beyond our expectations. Finally, we get a powerful endorsement of our research work from the user feedback and find out several promising directions. Note that while our system is able to construct travel route according to different personal start points, our experiments are just based on the same start point.

As the system needs the user to input some initial requirement, we assume united information for the experiments for the convenience of evaluation.

\section{Input Information}

Traveling city: Shenzhen, China

Traveling date: Nov, 20, 2013

Start point: Graduate School at Shenzhen, Tsinghua University

According to the initial information, the system gives three travel routes recommendation(see Fig. 4(a)(b)(c)). To explore the usefulness of the system, we design a survey questionnaire. This goal of this survey is to understand how users perceive our system-recommended route and the website-recommended route (see Fig. 4(d)) via direct comparison. Besides, we also want to explore the most meaningful and important information in the eyes of common travelers.

TABLE I: The DetaIls of DOUBan Event Metadata photo_id

TABLE II: THE DETAILS OF DOUBAN EVENT METADATA \begin{tabular}{|l|l|l|l|l|l|l|l|}
\hline title & time & address & latitude & longitude & type & participant & wisher \\
\hline
\end{tabular}

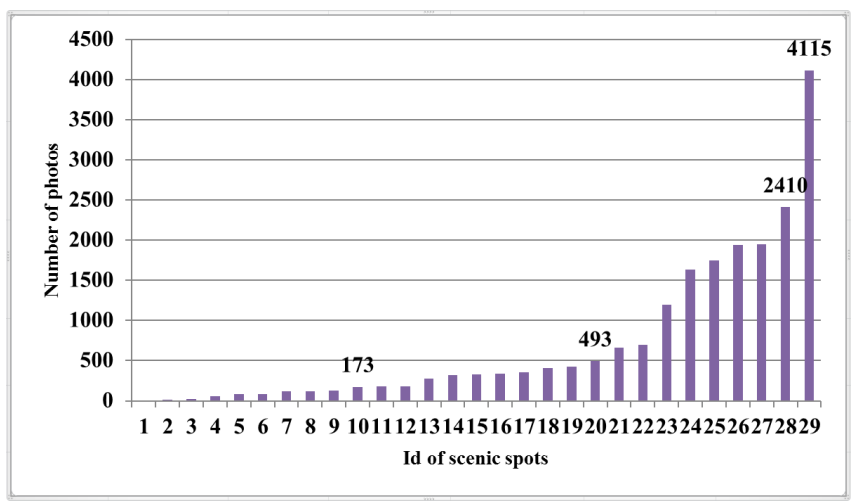

Fig. 3. The number of photos of scenic spots.

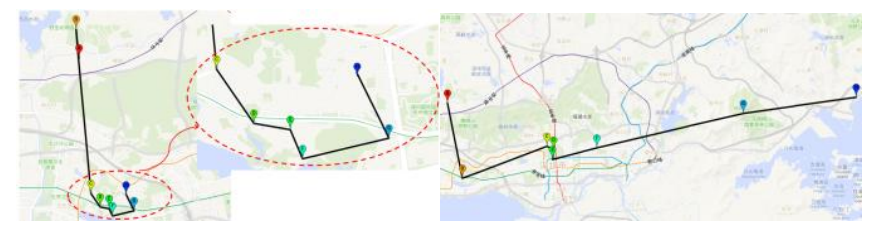

(a) DR approach

(b) PR approach

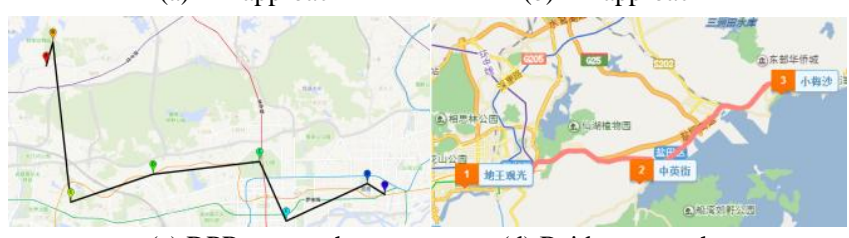

(c) DPR approach

(d) Baidu approach

Fig. 4. Recommended travel route.

Q1 and Q2 of the survey are selection of travel route and POIs, in which we ask the user to choose the best route and some desirable/undesirable POIs; Q3 is to explore whether the activities information are valuable to the user; Q4,5,6 are to inquiry user's attitude towards such travel recommendation app, which help us to understand the prospect of developing such app. Q7 is to investigate which functions are concerned by the user and they may become our future research directions. All the questions of the survey are demonstrated in Appendix B.

\section{Results analysis}

In this section, we describe the result of the above survey questionnaire and give analysis of the user feedback. A total 32 questionnaires are received and the result is depicted in Fig. 5.

From Q1, we get a positive feedback of our recommendation. Only two participates choose the route constructed by the travel website that is worse than any one of our recommendation routes. Apparently our system wins the vast majority of users' selection. Among the three items, the route TR_DR is the most popular and TR_DPR recommended by a blended method perform the worst, which is beyond of our expectation. We think there are two factors behind this phenomenon. One is that the user pays much 
attention to the transit time between various POIs. The less time wasted on the road, the happier the tourist feels. And the distance-based recommendation meets this need. The other is that we have filtered the unpopular POIs in the process of preparing dataset, thus the candidate POIs are generally popular. So the user prefers to visit several POIs in a smaller district.

In $\mathrm{Q} 2$, we delete the items that are only liked or disliked once (eg. Only one student likes Wutong Mountain and no one dislikes it, so we don't include it in our diagram here).We define a concept of 'pure interest' here.

$$
\text { pure interest }=N_{\text {likes }}-N_{\text {dislikes }}
$$

$N_{\text {likes }}$ and $N_{\text {dislikes }}$ stand for the number of likes and dislikes respectively. The POIs whose value of 'pure interest' is greater than zero are Happy Valley, China Folk Culture Villages, City of Sky, Shenzhen Safari Park, Dameisha, Splendid China, Jazz Concert. In these seven POIs, there are two activities and it also proves the importance of taking activities into account.

Q3 is designed to verify the usefulness of activities. The picture demonstrates that everyone shows interest in participating in local activities, which illustrates the necessity of bringing activities into the travel route once again. Meanwhile, most users say that the type of activities is vital and if it meets their tastes, they will consider attending it. We are inspired that we need to consider both the type of activities and users' preference in the future work in order to get better performance.

Through Q4,5,6, we know that over $87 \%$ volunteers regard these routes as a meaningful recommendation, only $6.25 \%$ participants declare they won't use such app and the users who would like to recommend such app to their friends account for more than $81 \%$. All this data prove the effectiveness of the recommendation system.

Q7 is posted to enrich the function of the recommendation system. The figure shows that the means of transportation and the visiting time in POIs are the most necessary information to consider. Besides, some users propose other demands: 1) distribution of car park; 2) wifi information near POIs; 3) feedbacks on the POIs from other tourists; 4) distribution of population based on time. All the above suggestions indicate the requirements of the tourists and may become our future research work.

\section{RELATED WORK}

Recommendation target: A brunch of work aims to simply recommend scenic spots or activities. Liu et al. propose a method to predict a user's favorite locations in a city [10]. Van Setten et al. gives a ranked list of activities according to the user's interest [11]. Based on these work, a travel route is provided by Liu et al. . Cao et al. make travel package recommendation which includes the topics (tour style), travel duration, price, area and attractions [1].

Recommendation models: The basic approaches to travel recommendation include content-based recommendation, collaborative recommendation and topic model. Yuan Jing et al. treat regions as documents and functions as topics [8].
Long et al. also take advantage of LDA model to discover the local geographic topic form the check-in record extracted from Foursquare [12]. Van Setten et al. adopt a blended model. Content-based recommenders make use of a direct matching between the tags of activities and the user interest towards the tags. Collaborative filtering recommender aims to find similar users by taking into account the demographic information and explicit ratings [11]. Yu et al. synergize content-based, Bayesian-classer and rule-based methods in their recommendations for smart phones [13].

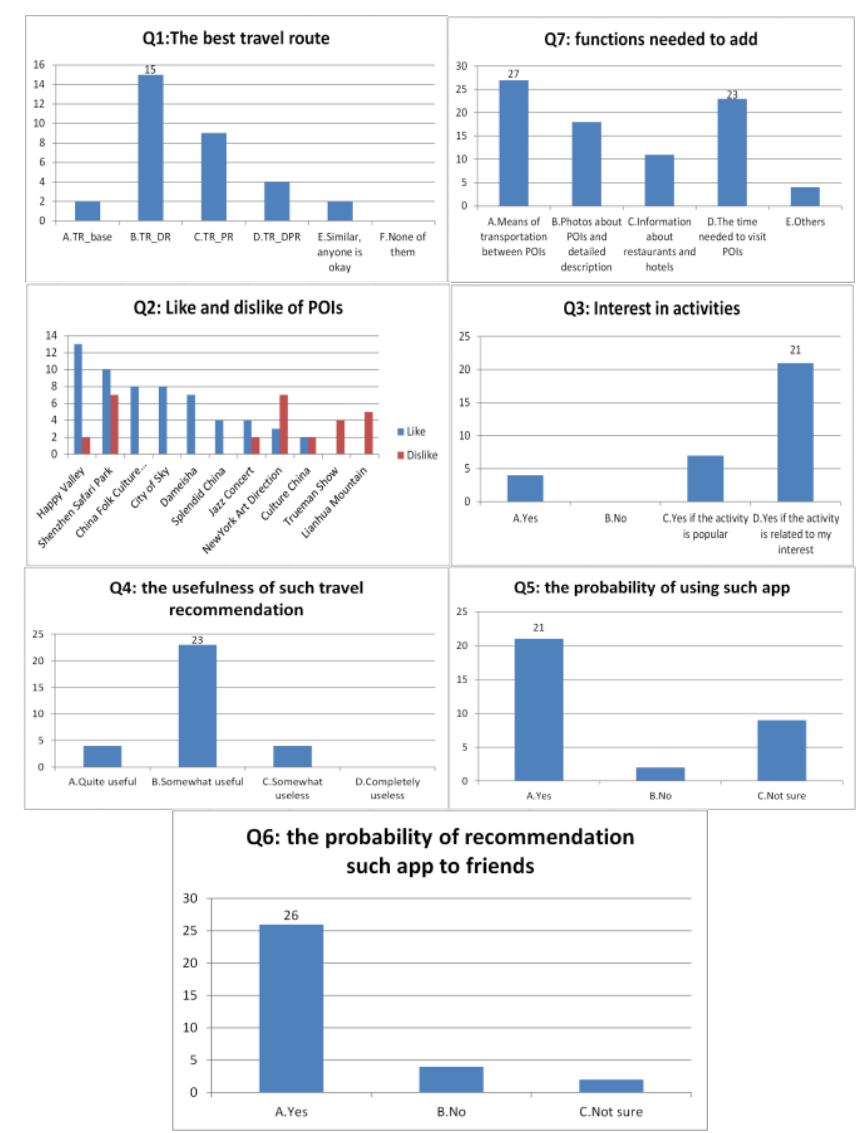

Fig. 5. Statistical result of the survey questionnaire.

\section{CONCLUSION}

This paper addressed the question of real-life travel route recommendation. The user input their tourism requirements, then the system make good use of POI information to get the popularity and geo-coordinates of them. Flickr provides the metadata of scenic spots and DoubanEvent is the source of activities information. We propose three rule-based methods to produce travel route recommendation and take experiments to evaluate the quality of these recommendations. The feedback of survey questionnaire yields promising result and distance-based recommendation lead to the best travel route. To the best of our knowledge, this is the first work that takes activities information into travel route recommendation together with traditional attractions information, which widely enlarges the choice of tourists visiting places.

In the future, we will further study how to build model for user profile so that we can understand the user's travel interests better. At the same time, we are going to consider the travel time, including the visiting time in every POI and 
the transit time from one place to another.

\section{APPENDIX}

A: ID-SCENIC SPOTS

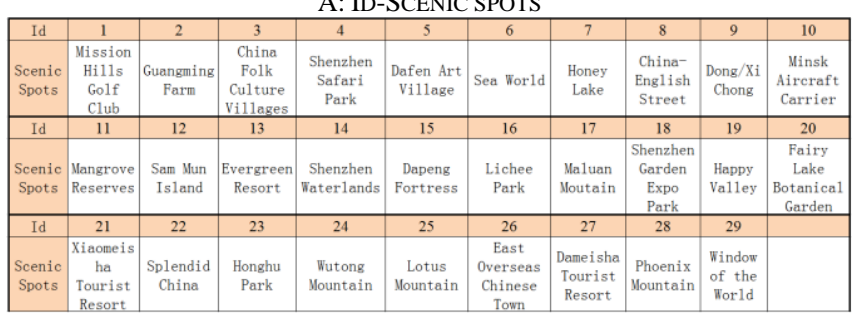

B: DESCRIPTION OF EVALUATION QUESTIONNAIRE

Q1: Which travel route interests you most in the candidate four choices?
A. TR_base
B. TR_DR
C. TR_PR
D. TR_DPR

E. Similar, anyone is okay F. None of them

Q2: For the route you are interested in, which POIs youlike and dislike particularly?

Like: Dislike:

Q3: If you travel to a city, would you be interested in local activities (such as concert/drama/show/party and so on) except visiting scenic spots?

$\begin{array}{lll}\text { A. Yes } & \text { B. No C. Yes if the activity is popular }\end{array}$

D. Yes if the activity is related to my interest

Q4: How would you rate this travel recommendation system

A. Quite useful B. Somewhat useful

C. Somewhat useless D. Completely useless

Q5: Would you like to use this travel assistant if such app is posted online?

A. Yes B. No C. sure

Q6: Would you like to recommend such app to your friends?

$\begin{array}{lll}\text { A. Yes } & \text { B. No C. Not sure }\end{array}$

Q7: Which functions would you like to add to such app? (multiple choice)

A. Means of transportation between POIs

B. Photos

about POIs and detailed description C. Information

about restaurants and hotels D. The time needed to

visit POIs E. Others:

\section{REFERENCES}

[1] L. Cao, J. Luo, A. Gallagher, X. Jin, J. Han, and T. S. Huang, “A worldwide tourism recommendation system based on geotagged web photos," in Proc. IEEE International Conference on Acoustics Speech and Signal Processing (ICASSP), 2010, pp. 2274-2277.

[2] Y. S. Reddy et al., "TripEneer: User-Based travel plan recommendation application," in Proc. 7th International AAAI Conference on Weblogs and Social Media, 2013, vol. 130.

[3] H. Yin, Y. Sun, B. Cui, Z. Hu, and L. Chen, "LCARS: A location-content-aware recommender system," in Proc. the 19th ACM SIGKDD International Conference on Knowledge Discovery and Data Mining, 2013, pp. 221-229.

[4] M. Clements, P. Serdyukov, A. P. De Vries, and M. J. Reinders, "Using flickr geotags to predict user travel behavior," in Proc. the 33rd International ACM SIGIR Conference on Research and Development in Information Retrieval , 2010, pp. 851-852.

[5] A. Majid, L. Chen, G. Chen, H. T. Mirza, I. Hussain, and J. Woodward, "A context-aware personalized travel recommendation system based on geotagged social media data mining," International Journal of
Geographical Information Science, vol. 27, no. 4, pp. 662-684, Jun. 2012.

[6] A. Popescu and G. Grefenstette, "Deducing trip related information from Flickr," in Proc. the 18th International Conference on World Wide Web, 2009, pp. 1183-1184.

[7] H. S. Chiang and T. C. Huang, "User-adapted travel planning system for personalized schedule recommendation," Information Fusion, 2013.

[8] J. Yuan, Y. Zheng, and X. Xie, "Discovering regions of different functions in a city using human mobility and POIs," in Proc. the 18th ACM SIGKDD International Conference on Knowledge Discovery and Data Mining, 2012, pp. 186-194.

[9] M. Antonio et al., "Sigtur/e-destination: ontology-based personalized recommendation of tourism and leisure activities," Engineering Applications of Artificial Intelligence, vol. 26, pp. 633-651, Jan. 2013

[10] Q. Liu, Y. Ge, Z. Li, E. Chen, and H. Xiong, "Personalized travel package recommendation," in Proc. IEEE 11th International Conference on Data Mining (ICDM), 2011, pp. 407-416.

[11] M. Van Setten, S. Pokraev, and J. Koolwaaij, "Context-aware recommendations in the mobile tourist application compass," Adaptive Hypermedia and Adaptive Web-Based Systems, pp. 235-244. Springer 2004.

[12] X. Long, L. Jin, and J. Joshi, "Exploring trajectory-driven local geographic topics in foursquare," in Proc. ACM Conference on Ubiquitous Computing, 2012, pp. 927-934.

[13] Z. Yu, X. Zhou, D. Zhang, C.-Y. Chin, X. Wang et al., "Supporting context-aware media recommendations for smart phones," IEEE Pervasive Computing, vol. 5, no. 3, pp. 68-75, July 2006

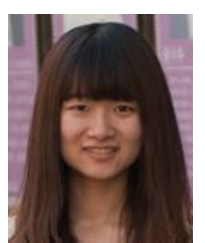

Jie $\mathbf{L i}$ received her BSc degree in electronic information engineering from Zhejiang University, Hangzhou, China in 2011. She is currently working toward the master degree in Automation Department of Tsinghua University.

Her research interests include recommendation system and machine learning.

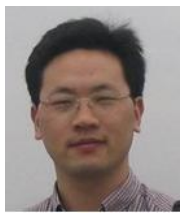

Yujiu Yang received the BSc degree in applied geophysics from China University of Mining Technology, Xuzhou, China in 1995; the MSc degree in control theory and control engineering from China University of Mining Technology in 2002 and the PhD degree in pattern recognition and intelligent systems from China Academy of Sciences, Institute of Automation in 2008.

Since 2010, he has been giving lectures in the Information Department of Graduate at Shenzhen, Tsinghua University, Shenzhen, China, where he currently is a lecturer. His current research interests include on information retrieval, Web data mining technology and application.

Mr. Yang instructed his students to win the fifth place in $13^{\text {th }}$ PAKDD international competition.

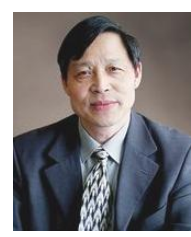

Wenhuang Liu received the $\mathrm{BSc}$ and $\mathrm{PhD}$ degree in the Automation Department from Tsinghua University, Beijing, China in 1970 and 1975 respectively. In 1986, he was a visiting scholar in Colorado University and did research on nonlinear control and robot vision

$\mathrm{He}$ is the professor in the Automation of Department, Tsinghua University. His research interests focuses on computer integrated manufacture system. He is an author or coauthor of more than 20 publications. 